This Section of Epidemiology and Psychiatric Sciences regularly appears in each issue of the Journal to cover methodological aspects related to the design, conduct, reporting and interpretation of clinical and epidemiological studies. The aim of these Editorials is to help developing a more critical attitude towards research findings published in international literature, promoting original research projects with higher methodological standards, and implementing the most relevant results of research in every-day clinical practice.

Corrado Barbui, Section Editor and Michele Tansella, Editor EPS

\title{
What is the WHO essential medicines list?
}

\author{
M. Purgato* and C. Barbui
}

Department of Public Health and Community Medicine, Section of Psychiatry, University of Verona, Verona, Italy

This article briefly presents the main characteristics of the World Health Organization (WHO) essential medicines list (EML), a register of minimum medicine needs for every health-care system. The idea behind the list is that the use of a limited number of well-known and cost-effective medicines may lead to improved long-term medicine supply, lower costs and better health care provision.

Received 6 June 2012; Revised 7 June 2012; Accepted 12 June 2012; First published online 30 July 2012

Key words: Essential list of medicines, mental health, psychotropic drugs, World Health Organization.

The World Health Organization (WHO), established in 1948 after the Second World War, is a specialized agency of the United Nations serving as the directing and coordinating authority for international health matters and public health (World Health Organization, 2009). One of WHO's constitutional functions is to offer objective and reliable information and advice in the field of human health. The organization seeks through its publications to support national health strategies addressing the most pressing public health concerns of populations around the world. To respond to the needs of Member States at all levels of development, WHO publishes practical manuals, handbooks and training material, guidelines, analyses of health policies and lists of medicines to treat priority health care conditions (World Health Organization, 2009).

The WHO essential medicines list (EML) is a register of minimum medicine needs for every health-care system (Bloom, 2011). The basic concept is that high

* Address for correspondence: Dr Marianna Purgato, Department of Public Health and Community Medicine, Section of Psychiatry, University of Verona, Piazzale L.A. Scuro, 10 - 37134 Verona, Italy.

(Email: marianna.purgato@univr.it) priority drugs should be available as part of a functioning health system at all times for all people, guiding physicians to evidence-based and rational prescribing. The idea behind the list is that the use of a limited number of well-known and cost-effective medicines leads to improved long-term medicine supply, lower costs, better health care and more equitable and sustainable access to products (Hutchings et al. 2010).

First published in 1977, the EML is revised every 2 years by a committee of experts from around the world in fields such as medicine, pharmacology, policy, regulation and health organizations (Hutchings et al. 2010). The expert committee updates the model list to reflect changes in global health concerns, pharmaceutical developments and patterns of drug resistance. The current version is the 17th WHO EML (updated in March 2011). The list is meant not to replace individual, national EML, but rather to be used as a guide to inform purchasing decisions in the context of national health agendas (Kishore \& Herbstman, 2009).

The current list contains over 350 drugs, organized by category of medication and identified by generic names. For mental and behavioural disorders, there 
are 12 compounds: amitriptyline and fluoxetine for depressive disorder; chlorpromazine, haloperidol and fluphenazine decanoate for psychotic disorder; carbamazepine, lithium carbonate and valproic acid for bipolar disorder; diazepam for anxiety disorder; clomipramine for obsessive-compulsive disorder; nicotine replacement therapy and methadone for disorders due to psychoactive substance use (World Health Organization, 2011a; Purgato et al. 2011). It should be noted that chlorpromazine, fluphenazine, haloperidol, amitriptyline and diazepam are indicated as an example of the class for which there is the best evidence for effectiveness and safety.

The procedure to create and revise the EML has changed substantially over time. Originally, selections largely involved decisions made by members of the expert committee, which were only partially based on the evidence. In 2002, in response to growing concerns about the methodology followed to update the list, an approach based on a more systematic assessment of the evidence base was adopted, which included careful appraisal of efficacy, safety, costeffectiveness and public-health relevance. Links with the Cochrane Collaboration were established in order to make better evidence-based decisions (Laing et al. 2003; Liberati, 2011).

Applications for inclusions, changes or deletions to the model list can be prepared by outside institutions, but have to be evidence-based and must explain why a specific drug meets the criteria of the model list for acceptance. WHO states that the selection process is based on factors such as disease prevalence, scientific evidence and comparative cost-effectiveness (Figure 1). Each of these factors contributes with a different weight to the decision process, depending on which kind of medicine is under evaluation for which condition.

It should be acknowledged that WHO has progressively improved the transparency of reporting the whole process, and currently the on-line availability of material regarding expert committees' meetings is a very relevant step forward. We argue, however, that the transparency in the reporting of the selection process might be further improved. In the field of psychotropic drugs, for example, out of 51 rejected pharmacological interventions 12 were psychotropic drugs (six antipsychotics, four antidepressants, one anticonvulsant and one benzodiazepine) (World Health Organization, 2011b). We note that the specific reasons for exclusion are not clearly reported using a standardized template, and this may create some degree of ambiguity. Clozapine for example - an atypical antipsychotic medication used in the treatment of schizophrenia - is mentioned and recommended in the recently issued mhGAP Intervention Guide (World Health Organization, 2011c), but it

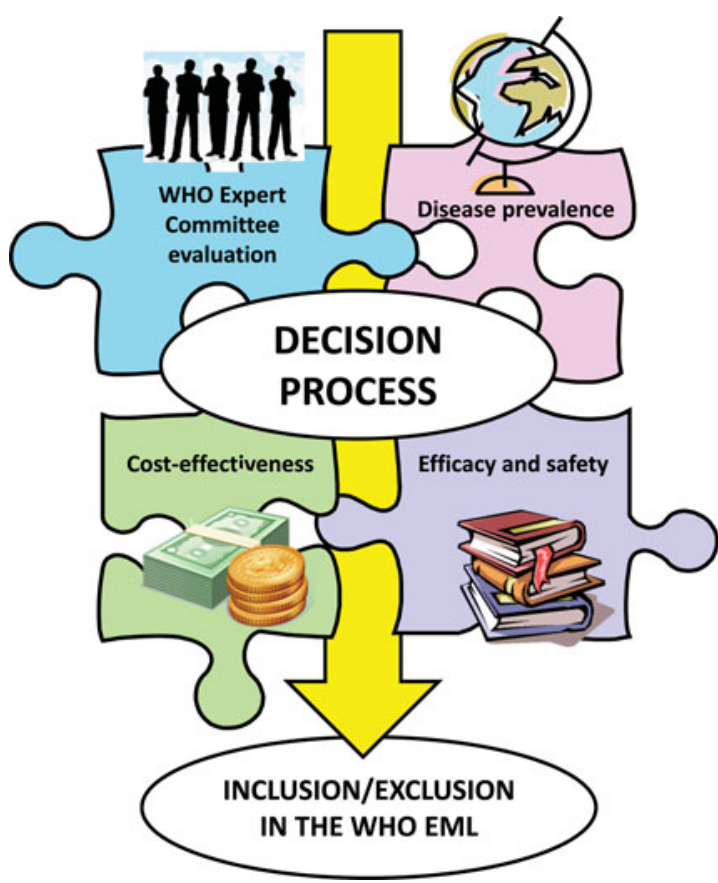

Fig. 1. Factors considered by the WHO in the selection of medicines for the WHO EML.

simultaneously appears in the WHO list of rejected drugs without a clearly reported reason.

Better provision for information on the WHO selection process would represent a major achievement. Policy-makers and health care professionals would have the opportunity to know the reasons for approval or rejection of newly submitted drugs, and consumers would have the chance to closely monitor the whole drug selection process aiming for continuous improvement.

\section{References}

Bloom BR (2011). WHO needs change. Nature 473, 143-145.

Hutchings J, Neroutsos K, Donnelly K (2010). Making the list: the role of essential medicines lists in reproductive health. International Perspectives on Sexual and Reproductive Health 36, 205-208.

Kishore SP, Herbstman BJ (2009). Adding a medicine to the WHO model list of essential medicines. Clinical Pharmacology and Therapeutics 85, 237-239.

Laing R, Waning B, Gray A, Ford N, Hoen E (2003). 25 years of the WHO essential medicines lists: progress and challenges. Lancet 361, 1723-1729.

Liberati A (2011). The Cochrane Collaboration as key player in the struggle for better and more relevant patients- and system-oriented research. Epidemiology and Psychiatric Sciences 20, 225-230.

Purgato M, Barbui C, Adams CE (2011). Using the needs of WHO to prioritise Cochrane reviews: the case of antipsychotic drugs. International Journal of Mental Health Systems 5, 25. 
World Health Organization (2009). The Selection and Use of Essential Medicines. WHO: Geneva. Retrieved 5 June 2012 from http://whqlibdoc.who.int/trs/WHO_TRS_958_eng.pdf World Health Organization (2011a). Model List of Essential Medicines. WHO: Geneva. Retrieved 5 June 2012 from http://whqlibdoc.who.int/hq/2011/a95053_eng.pdf

World Health Organization (2011b). WHO List of rejected drugs from the list of Essential Medicines. WHO: Geneva.
Retrieved 5 June 2012 from http://www.who.int/ selection_medicines/committees/REJECTIONS.pdf World Health Organization (2011c). mhGAP Intervention Guide for mental, neurological and substance use disorders in non-specialized health settings. WHO: Geneva. Retrieved 5 June 2012 from http://whqlibdoc. who.int/publications/2010/9789241548069_ eng.pdf 